\title{
Em busca de uma assistência adequada à saúde do idoso: revisão da literatura e aplicação de um instrumento de detecção precoce e de previsibilidade de agravos
}

\author{
The quest for adequate health care for the elderly: \\ literature review and the application of an \\ instrument for early detection and prediction \\ of diseases
}

Renato Veras 1,2

\footnotetext{
1 Instituto de Medicina Social, Universidade do Estado do Rio de Janeiro. Rua São Francisco Xavier 524, 7o andar, Rio de Janeiro, $R J$ 20559-900, Brasil. 2 Universidade Aberta da Terceira Idade, Universidade do Estado do Rio de Janeiro. Rua São Francisco Xavier 524, 10 o andar, Rio de Janeiro, $R J$ 20559-900, Brasil.
}

\begin{abstract}
The increase in the elderly population demands changes in health care models by policymakers to deal with the substantial cost increase This article addresses patient access flow and the definition of priorities in an outpatient unit. A short questionnaire (8 questions) was used to assess enrollment and ranking of the elderly at risk of repeated hospital admissions, considered as a proxy for frailty. In a sample of 360 individuals, $75.8 \%$ of the elderly population was classified as "low risk". However, approximately $11 \%$ of the sample was considered to be at "medium" or "high" risk of becoming ill and requiring intensive use of health services. Our findings corroborate the usefulness (for promoting an effective health system for the elderly) of the proposed questionnaire as a tool to classify the elderly in different "frailty groups", using a hierarchic definition of vulnerability. The proposed ranking procedure can help adjust the portal of entry into the system, as used thus far in most Brazilian health facilities. The article concludes by suggesting that the validity of the method be tested in a prospective study.
\end{abstract}

Key words Elderly Population; Screening Procedures; Frailty; Health Planning; Public Health

Resumo O aumento da população idosa exige dos gestores da área da saúde mudanças nos modelos assistenciais, em decorrência da significativa ampliação dos respectivos custos. Este artigo discute uma proposta de fluxo de acesso e definição de prioridades em uma rede ambulatorial. Utilizou-se um questionário breve, com oito perguntas objetivas, visando à captação e ordenação de idosos em risco de admissão hospitalar repetida, tomada como proxy de fragilização. Em uma amostra de 360 indivíduos, evidenciou-se que 75,8\% dos idosos apresentavam baixo risco de adoecer. Cerca de $11 \%$ da amostra, contudo, apresentou risco de médio a alto de adoecer, utilizando de forma intensiva os serviços de saúde. Os achados corroboram a utilidade do procedimento proposto a fim de classificar idosos em diferentes grupos de risco de fragilização, possibilitando a hierarquização dos riscos nessa população e contribuindo para um sistema de saúde mais eficaz e resolutivo. Sugere-se testar a validade do método em um estudo prospectivo.

Palavras-chave Idosos; Instrumentos de Detecção Precoce; Fragilização; Planejamento de Saúde; Saúde Coletiva 


\section{Introdução}

A espécie humana necessitou de milhões de anos para atingir um bilhão de pessoas, o que teria ocorrido provavelmente em 1830. Em 1927 este número dobrou. Em 1960, a população mundial chegou aos três bilhões de habitantes. De lá para cá, esse incremento populacional experimentou substancial aceleração. Em 14 anos a marca dos quatro bilhões foi atingida, o quinto bilhão veio em 1987 e, 12 anos depois, em 1999, alcançamos o sexto bilhão. Paralelamente ao aumento populacional, estendeu-se a longevidade humana a limites antes impensados.

O Brasil é um país que envelhece a passos largos. As alterações na dinâmica populacional são claras, inexoráveis e irreversíveis. No início do século 20 , um brasileiro vivia em média 33 anos, ao passo que hoje a expectativa de vida dos brasileiros atinge os 68 anos (Ramos, 1993; Silvestre, 1998.). Entre 1960 e 1980, observouse no Brasil uma queda de $33 \%$ na fecundidade (Alves, 1997; Veras, 2001). A diminuição no ritmo de nascimento resulta, a médio prazo, no incremento proporcional da população idosa. Nesse mesmo período de 20 anos, a expectativa de vida aumentou em oito anos. Hoje, a população de idosos ultrapassa os 15 milhões de brasileiros (para uma população total de cerca de 170 milhões de habitantes), que em 20 anos serão 32 milhões (Veras, 2002).

Nunca antes na história da humanidade os países haviam registrado um contingente tão elevado de idosos em suas populações. Para se ter uma noção da magnitude desse crescimento, basta verificar a população de certas áreas, como, por exemplo, e em especial, a do bairro de Copacabana, no Rio de Janeiro, com $26 \%$ de idosos, uma proporção superior a países com população que conta há muito com uma importante parcela de iodos (Veras et al., 2002).

É chegado o momento de ir além da descrição, basicamente quantitativa, das mudanças do perfil demográfico para uma reflexão na qual as mudanças culturais e sociais, decorrentes dessa ampliação, estejam mais presentes. As repercussões das profundas transformações sociais decorrentes do envelhecimento ainda são pouco contempladas, cabendo observar que esse é o início de um processo em curso. O envelhecimento populacional se ampliará em decorrência dos avanços nos conhecimentos da engenharia genética e da biotecnologia, alterando em um futuro próximo não apenas indicadores demográficos como a expectativa de vida, mas principalmente o próprio limite do tempo de vida, ou relógio biológico (Fries, 1980; Fries \& Crapo, 1981). Nos dias atuais, o relógio biológico da espécie humana atinge 90-95 anos (Veras, 2001; Veras et al., 2002). Estima-se, no entanto, que nas próximas décadas esse indicador se ampliará, alcançando 120-130 anos. O desafio que se apresenta é a elaboração de cenários em que os avanços da ciência e da tecnologia permitirão ao ser humano alcançar esses limites de forma independente, não fragilizado, livre de diversas doenças e com uma expectativa de vida que se aproxime do limite biológico máximo.

Teremos indivíduos se aposentando aos 60 anos e iniciando um novo ciclo de trabalho por mais 30 ou 40 anos. Na área da educação teremos possivelmente formação profissional e cursos universitários especificamente para cidadãos de mais de 60 anos. A razão mulheres: homens será provavelmente ainda maior, e a proporção de mulheres viúvas e vivendo sós deverá aumentar, com conseqüências ainda pouco avaliadas. Os novos medicamentos poderão debelar muitas doenças, e outra grande mudança, em parte decorrente dos avanços dos fármacos, dar-se-á na esfera da sexualidade, com a introdução de medicamentos que permitirão uma vida sexual ativa substancialmente ampliada. É possível que tenhamos, em breve, famílias com cinco gerações, algumas delas às voltas com o desemprego, devido ao processo de globalização, cabendo aos mais idosos o papel de sustentáculo, por contarem com aposentadoria e terem conseguido reinserção no mercado de trabalho (Camarano, 1999a, 1999b).

Ao propor uma mudança do patamar de discussão da transição demográfica, deve-se buscar algo análogo na área da saúde, em relação à (re)organização dos modelos assistenciais. Precisamos responder a esses desafios de modo propositivo. É intenção deste trabalho contribuir para a discussão de tais questões, revisando criticamente aspectos centrais da literatura e formulando e testando uma proposta de acesso mais equânime aos serviços de saúde.

\section{As doenças passam a ser crônicas e múltiplas}

O aumento dos idosos na população implica, em termos de utilização dos serviços de saúde, um maior número de problemas de longa duração, que freqüentemente exigem intervenções custosas, envolvendo tecnologia complexa para um cuidado adequado (Veras, 1994, 2001). Em menos de 40 anos, o Brasil passou de um perfil de mortalidade típico de uma população jovem para um quadro caracterizado por enfermidades complexas e onerosas, próprias das 
faixas etárias mais avançadas (Gordilho et al., 2000). Esse fato acarreta crescimento das despesas com tratamentos médicos e hospitalares, ao mesmo tempo em que apresenta um desafio para as autoridades sanitárias, especialmente no que tange à implantação de novos modelos e métodos de planejamento, gerência e prestação de cuidados (Veras, 2000). A baixa resolutividade do modelo em curso, a precariedade dos serviços ambulatoriais, a escassez dos serviços domiciliares, a falta de instâncias intermediárias (como os hospitais-dia e centros de convivência), fazem com que o primeiro atendimento ocorra em estágio avançado, dentro do hospital, o que não só aumenta os custos como diminui as chances de um prognóstico favorável (Mendes, 2001). Em geral, as doenças dos idosos são crônicas e múltiplas, perduram por vários anos e exigem acompanhamento médico constante e medicação contínua. Além disso, a abordagem médica tradicional, focada em uma queixa principal, e o hábito médico de reunir as queixas e os sinais em um único diagnóstico, podem ser adequados ao adulto jovem, mas não ao idoso.

\section{Indicadores de saúde}

Em um estudo de 1998, relativo ao acesso e à utilização dos sistemas de saúde, foram analisados vários indicadores de saúde (IBGE, 2000). Confirmou-se o que empiricamente já se sabia: o grupo etário acima dos 60 anos apresenta invariavelmente índices maiores de morbidade quando comparados aos demais grupos etários (Veras, 2001; Veras \& Alves, 1994). A morbidade referida e observada foi maior entre os idosos, assim como a auto-avaliação do estado de saúde foi pior entre os mais velhos. Observou-se também uma maior proporção de restrições de atividades habituais nos 15 dias anteriores à entrevista, por motivo de saúde, entre os de mais de 60 anos. Em relação às doenças crônicas, os idosos apresentaram números mais expressivos, quando comparados às demais faixas etárias. Pelo fato de a presença de patologias múltiplas, particularmente crônicas, ser maior entre os idosos, o número de exames complementares, quer laboratoriais ou de diagnóstico por imagens, também é superior entre os idosos (Vilar, 2000). A literatura brasileira recente (Mosegui et al., 1999; Rozenfeld, 1997; Sayd et al., 2001) aponta consistentemente para um maior uso de medicamentos entre os idosos. Em síntese, qualquer que seja o indicador de saúde analisado, haverá uma proporção maior de agravos e procedimentos médicos entre aqueles de mais de 60 anos, em comparação aos demais grupos etários, implicando maior utilização dos serviços de saúde e custos mais elevados.

\section{A "desospitalização" e os custos da saúde}

A discussão sobre "desospitalização” no Brasil começa a ganhar corpo (Brasil, 2002). Em 2000, dois artigos foram publicados em órgãos da imprensa sobre a questão. A revista Exame publicou uma matéria do Dr. Afonso José de Matos, coordenador do curso em gestão de saúde do IBMEC Business School (Matos, 2000), secundada por artigo do jornal $O$ Globo, do articulista Joelmir Beting (Beting, 2000). Em ambos os artigos, discute-se o fenômeno de aumento da oferta de número de leitos hospitalares no Brasil, em contraste com a tendência mundial da redução da oferta desses serviços. Encontra-se embutida nesse debate a questão dos custos crescentes do setor saúde, no qual ocorre uma situação considerada um "paradoxo tecnológico". Enquanto em todos os ramos da economia a incorporação de novas tecnologias vem reduzindo custos e melhorando produtos e serviços, na saúde, produtos e serviços ficam cada vez mais caros, sem que necessariamente melhore a qualidade do processo, ou seja, no caso da saúde, a cura e a melhora efetiva da qualidade de vida.

A possível justificativa para tal aumento pode ser imputada à crescente utilização dos espaços hospitalares para a instalação dos modernos e sofisticados equipamentos de exames diagnósticos. Caso essa tendência se amplie, observar-se-á uma situação inversa àquela observada nos países desenvolvidos, onde há uma transferência progressiva desses procedimentos para locais fora da unidade hospitalar, desafogando-a e tornando-a um espaço para intervenções específicas (como cirurgias mais complexas) e ações em determinadas patologias, sempre pelo menor tempo possível.

\section{O profissional de saúde que assiste o idoso}

Pelo fato de lidarmos com um grupo etário que tem crescido muito em anos recentes, existe uma enorme carência de profissionais treinados, com formação específica e cursos reconhecidos pela qualidade acadêmica. Como exemplo emblemático, a Sociedade Brasileira de Geriatria e Gerontologia (SBGG) reconhece pouco mais de 500 médicos como especialistas em geriatria, ou seja, profissionais que prestaram 
exame para obter o título de especialista, quando nos dias atuais a população de idosos ultrapassa 15 milhões de brasileiros. É evidente que excelentes médicos atendem idosos sem serem portadores da certificação da sociedade, mas a relação de 15 milhões para 500 não pode ser desconsiderada. Um exemplo que se observa, com freqüência, no mercado assistencial é o de médicos com idade mais avançada, que possuem uma clientela fiel que os acompanha há décadas, e que, portanto, vai progressivamente envelhecendo. Dessa forma, tais profissionais prestam assistência a uma população composta basicamente por idosos, não por opção ou formação específica, mas pelo fato de médico e clientela terem envelhecido juntos.

Os modelos a serem propostos para assistência ao idoso devem estar baseados nesta realidade assistencial - a carência de médicos especialistas em idosos. Ou seja, o profissional a ser utilizado prioritariamente não deverá ser o geriatra, pois este praticamente inexiste. A assistência deverá ser exercida pelo médico clínico, reservando apenas para casos bem definidos e criteriosamente selecionados o atendimento do geriatra. Esse clínico geral - para que se possa exigir dele alta resolutividade e baixo custo - precisará de instrumentos, particularmente aqueles que permitam a identificação mais correta possível dos distúrbios, a fim de que ele seja capaz de saber como tratar e quando encaminhar para o profissional com treinamento específico em envelhecimento humano.

\section{Fragilização e probabilidade de agravos}

Conforme mencionado, a população idosa é a que proporcionalmente consome mais serviços de saúde. No entanto, o nosso sistema de atenção precário e desorganizado não cria as condições para que esses recursos sejam utilizados melhor e mais adequadamente. Não que com isso os gastos em saúde com os idosos deixem de ser mais elevados nos países mais desenvolvidos; contudo, são aplicados de forma mais judiciosa e com maior resolutividade.

Por exemplo, nos Estados Unidos, é grande o volume de recursos empregados na tentativa de criar modelos de operação do sistema de saúde que encontrem uma conjunção ideal, otimizada, entre a qualidade dos serviços prestados e a quantidade de recursos despendidos. A lógica subjacente a esses estudos norte-americanos é de que a pequena parcela da população idosa que utiliza intensivamente os recursos destinados à saúde (naquele país, 5\% da população idosa registrada no Medicare é res- ponsável por $62 \%$ das despesas hospitalares) poderia gozar de melhor saúde e gastar menos em hospitalizações e recursos de complexidade tecnológica crescente se seus membros em maior risco fossem identificados precocemente e abordados de forma preventiva (Boult et al., 1993; Gruenberg et al., 1989).

$\mathrm{Na}$ busca de características preditivas de um uso intensivo de recursos de saúde, ou seja, na tentativa de identificar fatores de risco associados ao desenvolvimento de doenças graves, Boult et al. (1993) utilizaram um indicador - o risco de futuras hospitalizações -, que se mostrou significativamente relacionado a uma maior incidência cumulativa de readmissões hospitalares, a um número maior de dias de hospitalização entre os sobreviventes e a gastos aumentados durante o período de internação. Esses autores, mediante uma abordagem crítica de estudos anteriores e uma análise multivariada por meio de regressão logística, chegaram a oito variáveis que mostraram maior poder preditivo com relação à chance de admissão hospitalar (Boult et al., 1993). Com o auxílio de um breve questionário com oito perguntas, poder-se-iam identificar idosos em alto risco de serem hospitalizados duas ou mais vezes em um período de quatro anos, passando-se, portanto, a dispor de um instrumento confiável para a identificação de idosos fragilizados, candidatos a intervenções preventivas de saúde. Ainda segundo os mesmos autores, muitos desses idosos de "alto risco" identificados pelo procedimento de triagem (exceção feita àqueles com doenças terminais ou condições médicas instáveis), seriam beneficiados com programas preventivos, desenvolvidos em unidades de avaliação e reabilitação geriátricas (Boult et al., 1993).

A partir da proposta de Boult et al. (1993) imaginamos estruturar, focalizando a porta de entrada de um serviço público de saúde pertencente a uma universidade pública, localizada no Rio de Janeiro, com grande demanda da população idosa, uma lógica assistencial que priorizasse os que mais necessitam de cuidados (Tabela 1). A proposta foi a de estruturar um método de ordenação de prioridade desde o primeiro momento, ou seja, desde a entrada do idoso no sistema de saúde.

\section{Instrumento de identificação: a Triagem Rápida de Riscos}

O instrumento utilizado para a triagem e hierarquização de idosos em risco baseou-se nos estudos de Boult e colaboradores (Boult et al., 
1993; Boult et al., 1994; Pacala et al., 1995) (Tabela 2). Em nossa proposta, aceitamos o pressuposto básico desses autores de que, ao estimar a chance de internação repetida de uma determinada população, estamos avaliando a presença de um perfil de fragilização, que, além de aumentar a probabilidade de internações repetidas, leva à redução progressiva da capacidade funcional e, conseqüentemente, à maior demanda por serviços de saúde em todos os níveis.

Os fatores de risco considerados foram: idade, sexo, disponibilidade de cuidador, autopercepção da saúde, presença de doença cardíaca, presença de diabetes mellitus, pernoite hospitalar e consultas médicas nos últimos 12 meses.

O ponto de corte originalmente proposto por esses autores para a identificação de idosos em maior risco (fragilização $\geq 0,5$ ), embora útil no estabelecimento de estratos para intervenções únicas, não nos permitiria a flexibilidade necessária aos nossos propósitos. Em nosso estudo-piloto, a definição de um único ponto de corte não nos proporcionaria a informação necessária para a ordenação de prioridades de atendimento. Portanto, foram definidos novos pontos de corte, na tentativa de discriminar, de forma mais eficiente, menor e maior risco. Os pontos de corte foram escolhidos de forma arbitrária, a partir dos trabalhos dos autores. Entendemos, porém, que uma vez que o questionário é capaz de identificar indivíduos com doenças graves ou em risco ampliado de desenvolvê-las, a definição de uma linha de valores ordenados, com sucessivos pontos de corte, permitiria a definição daqueles em maior ou menor risco. É possível, a nosso ver, pressupor tal progressividade, de aumento concomitante de riscos e chances de demandar cuidados intensivos de saúde. Os limites propostos são descritos no Tabela 1.

\section{Fluxo de atendimento}

Os usuários de risco 0 serão cadastrados e convidados a retornar ao serviço dentro de três meses para nova avaliação, já que no momento da avaliação não apresentaram um perfil de risco que justificasse uma ação imediata. Caso o paciente tenha uma demanda específica (por exemplo, uma virose), esta deverá ser atendida diretamente, sem entrar no fluxo da consulta geriátrica.

Para os idosos identificados como de risco 1, 2 ou 3 (respectivamente riscos médio, médioalto e alto), propõe-se um fluxo de atendimento que se inicia na marcação de consulta ambulatorial com um clínico, precedida dos pro-
Tabela 1

Estratos de risco de fragilização.

\begin{tabular}{lcl}
\hline Nível & Risco de fragilização & Estratificação de risco \\
\hline Risco 0 & $<0,300$ & Baixo \\
Risco 1 & $0,300-0,399$ & Médio \\
Risco 2 & $0,400-0,499$ & Médio-alto \\
Risco 3 & $\geq 0,500$ & Alto \\
\hline
\end{tabular}

cedimentos iniciais de avaliação funcional, tal como delineado nos trabalhos referente à avaliação funcional (Veras, 2001). A avaliação funcional e todos os demais procedimentos do fluxo do cuidado ao idoso ultrapassam o escopo do presente trabalho. No entanto, as etapas necessárias para a organização desse serviço podem ser observadas na Figura 1. As fases sombreadas da figura deverão integrar um estudo futuro, restringindo-se o presente trabalho à etapa zero, ou seja, o processo de captação da clientela em um centro de saúde.

\section{Resultados}

\section{Amostra}

A amostra deste estudo foi composta por 360 indivíduos, entrevistados no guichê de recepção de um ambulatório da rede pública, tratando-se, portanto, de amostra não-aleatória (de conveniência), comum a estudos desta natureza, mas com reconhecidas limitações quanto à sua validade externa. Na amostra observase uma característica comum desta população em relação à demanda por serviços ambulatoriais: o percentual de homens é consistentemente menor que o de mulheres. Neste trabalho, dos 360 questionários analisados, 65,0\% (n =234) eram do sexo feminino.

Em relação à idade, estratificada no instrumento em quatro faixas, encontramos a seguinte distribuição: $66,1 \%$ com 65 a 74 anos; $22,8 \%$ com 75 a 79 anos; $8,3 \%$ com 80 a 84 anos; e 2,8\% com 85 anos ou mais. Os dados podem ser vistos na Figura 2. A estratificação da faixa etária difere um pouco da citada nos trabalhos de Boult e colaboradores, uma vez que foi necessário adaptar o instrumento para utilização em nosso meio, no qual, para fins de atendimento, alguns consideram idoso como aquele indivíduo com 65 anos ou mais, ainda que em desacordo com o preconizado nas diretrizes legais 
Tabela 2

Instrumento para triagem rápida.

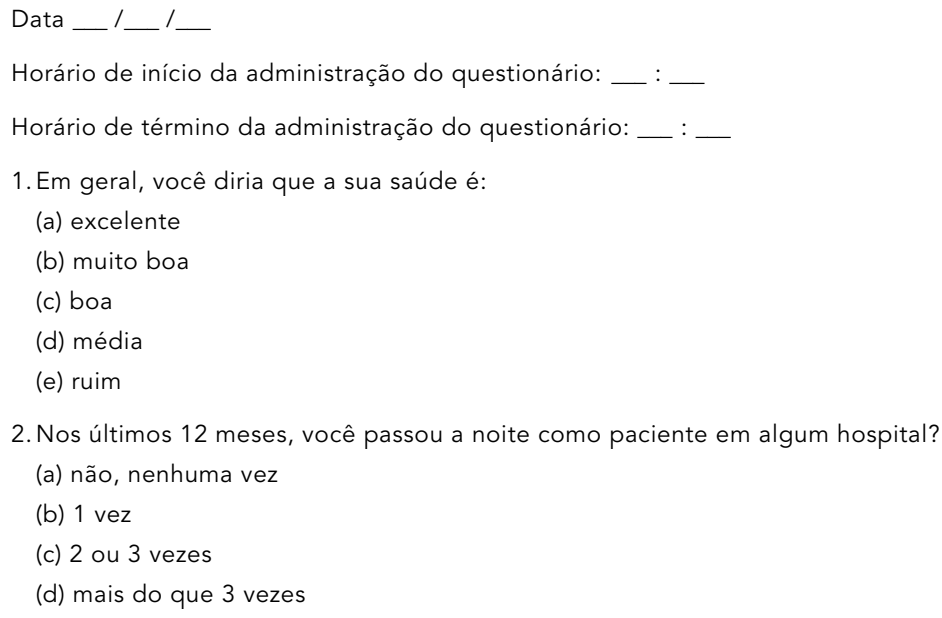

2. Nos últimos 12 meses, você passou a noite como paciente em algum hospital?
(a) não, nenhuma vez
(b) 1 vez
(c) 2 ou 3 vezes
(d) mais do que 3 vezes

3. Nos últimos 12 meses, quantas vezes você procurou o médico ou uma clínica para tratamento?

(a) nenhuma vez

(b) 1 vez

(c) 2 ou 3 vezes

(d) 4-6 vezes

(e) mais do que 6 vezes

4. Nos últimos 12 meses, você teve diabetes?
(a) $\operatorname{sim}$
(b) não

5. Você alguma vez já teve doença coronariana? [sim/não]

Angina? [sim/não]

Infarto do miocádio? [sim/não]

Ataque do coração? [sim/não]

(a) $\operatorname{sim}$ (se qualquer das respostas acima for "sim")

(b) não (se todas as respostas às questões acima for "não")

6. Sexo?
(a) Masculino
(b) Feminino

7. Existe algum amigo, parente ou vizinho que poderia tomar conta de você por alguns dias se você precisar?

(a) $\operatorname{sim}$

(b) não

8. Qual a sua data de nascimento?
(dia/mês/ano):
(a) 65-74 anos
(b) $75-79$ anos
(c) 80-84 anos
(d) $\geq 85$ anos

9. Nos últimos 4 anos você esteve internado mais de duas vezes?
(a) $\operatorname{sim}$
(b) não
(c) não se lembra

da Política Nacional do Idoso (Brasil, 1996; Gordilho et al., 2000), que define como idosos os indivíduos de 60 anos ou mais. Apesar de ter sido utilizado o ponto de corte de 65 anos, os dados mostram a necessidade de intervenção entre idosos mais jovens, que compõem a maior parte de nossa população idosa.

\section{Distribuição dos fatores de risco}

Entre os 360 entrevistados, cerca de $50 \%$ consideraram que a sua saúde era média, contrastando com $8,3 \%$ que consideravam ter uma saúde excelente ou muito boa. O fator de risco “internação nos últimos 12 meses" foi pouco mencionado nessa amostra, na qual $13,3 \%$ relataram ter estado internados ao menos uma vez no ano anterior. A freqüência de consultas médicas foi elevada, com $58,6 \%$ da amostra reportando quatro ou mais consultas nos 12 meses anteriores à entrevista.

A presença/diagnóstico de diabetes mellitus nos últimos 12 meses - um dos fatores de risco para repetidas internações nos trabalhos de Boult et al. $(1993,1994)$ - foi mencionada por aproximadamente $25 \%$ da amostra, enquanto as doenças cardiovasculares pesquisadas foram referidas por $35,1 \%$ dos indivíduos entrevistados.

A proporção de idosos que contavam com algum tipo de apoio social foi elevada, perfazendo $80,6 \%$ do total da amostra. Não foram observadas diferenças significativas desses fatores de risco em relação ao sexo do entrevistado. Os dados encontram-se sumarizados na Tabela 3 .

\section{Estratificação de risco}

Os idosos foram identificados e estratificados segundo o risco de internações repetidas, calculado a partir das respostas ao questionário aplicado. Em conformidade com outros estudos, os achados mostram que $75,8 \%$ da população apresenta baixo risco de adoecer. Cerca de $11 \%$ da amostra, contudo, apresentou risco de médio a alto de adoecer, necessitando utilizar os serviços de saúde de forma mais intensiva.

Ao relacionarmos o risco de internações repetidas com o sexo, não encontramos uma relação causal forte entre o risco de adoecer e o sexo do indivíduo, apesar de haver uma tendência de maior risco para o sexo masculino. A análise de risco mostrou um risco relativo de 1,19 (IC 95\%: 0,82-1,73) para os homens e de 0,84 (IC 95\%: 0,58-1,22) para as mulheres. 
Figura 1

Diagrama do fluxo de atendimento.

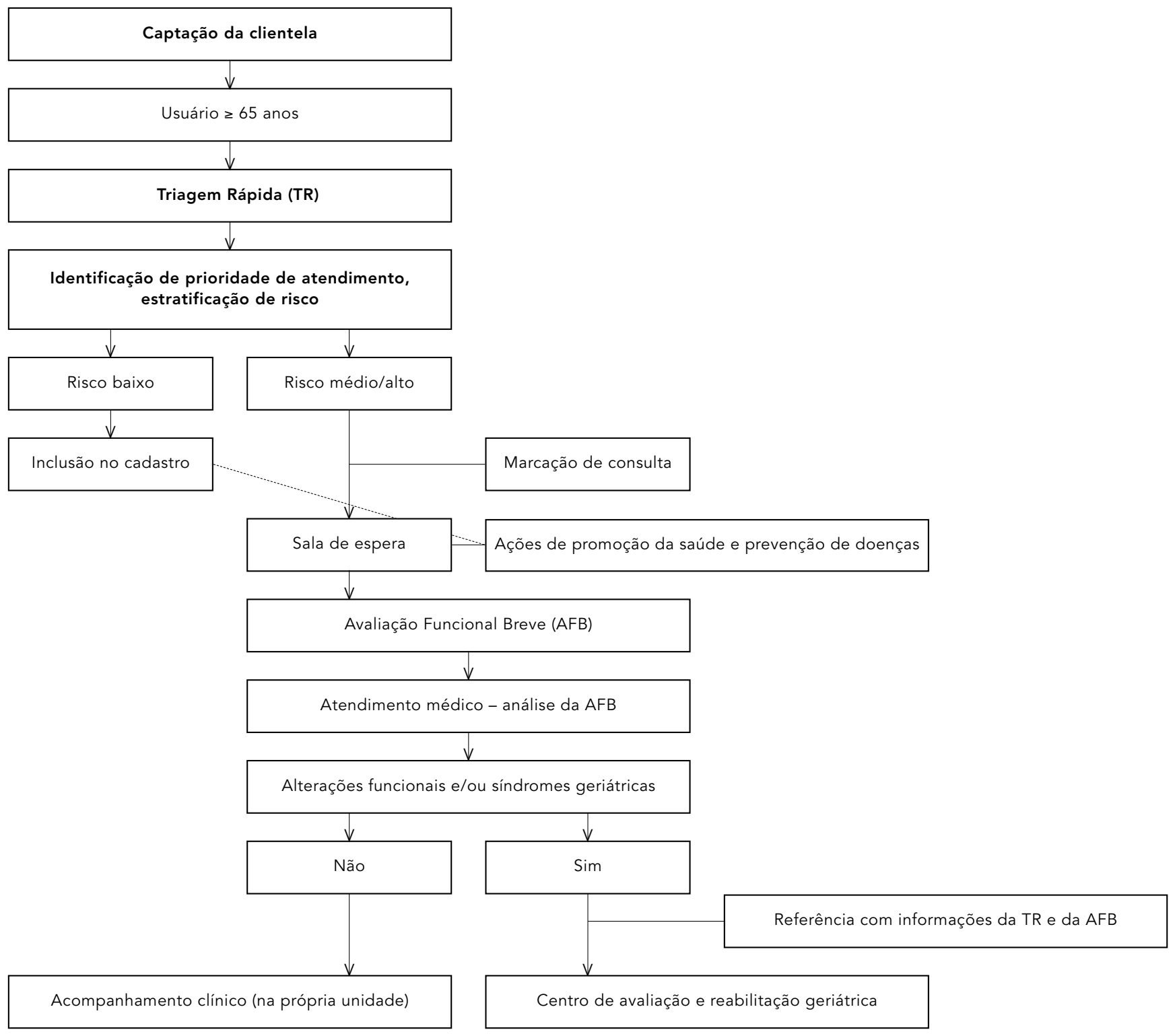

Fórmula logística para estimar a probabilidade de internação repetida (usada como proxy de fragilização):

Pra $=\frac{\mathrm{e}^{\mathrm{BX}}}{1+\mathrm{e}^{\mathrm{BX}}}$

onde: Pra $=$ probabilidade de internação repetida (fragilização); $\mathrm{E}=$ logaritmo natural; $\mathrm{BX}=\mathrm{B}_{0}+\mathrm{B}_{1} \mathrm{X}_{1}+\mathrm{B}_{2} \mathrm{X}_{2}+\ldots \ldots \ldots \mathrm{B}_{13} \mathrm{X}_{13}$;

$B_{0}=$ constante da equação de regressão logística $(-1,802) ; X=1$ ou 0 , presença ou ausência de cada fator de risco;

$B=$ coeficiente da regressão logística de cada fator de risco. 
Figura 2

Distribuição dos idosos, por sexo e faixa etária.

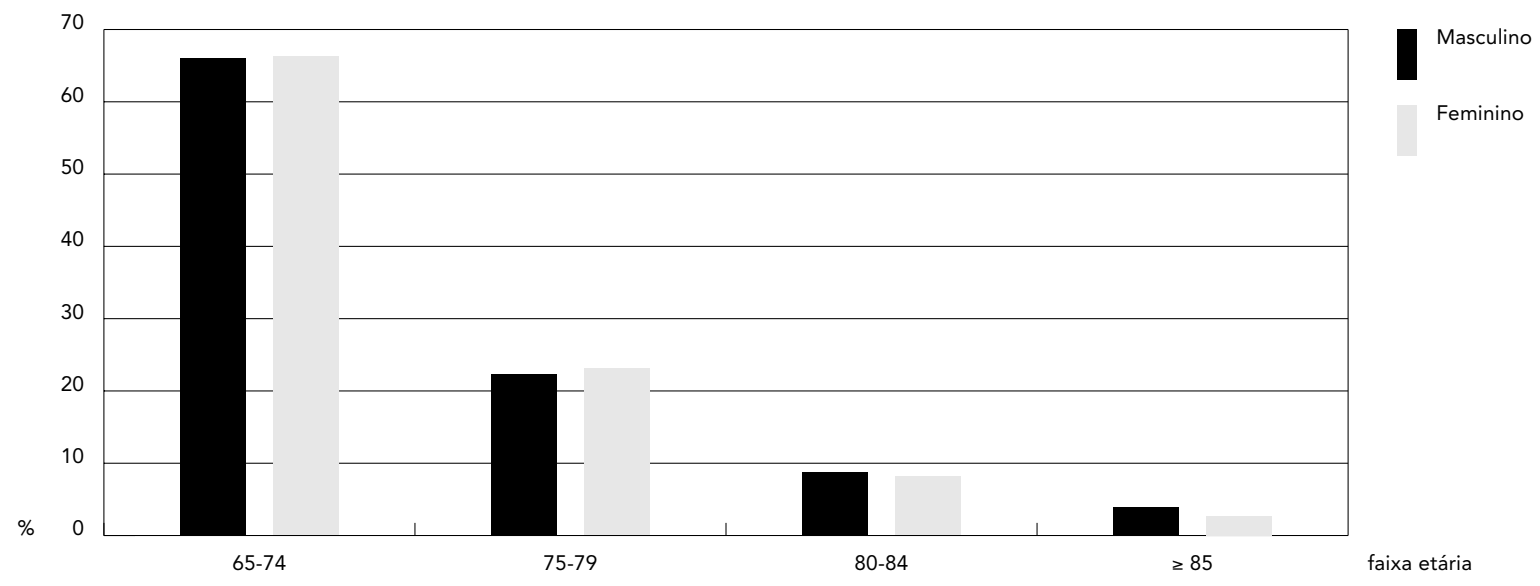

Em relação à idade, o risco mostrou-se levemente maior e estatisticamente significativo para os indivíduos de idade mais avançada (80 anos ou mais), em parte devido ao seu pequeno número na amostra. A pergunta sobre hospitalização nos últimos 12 meses guarda estreita relação com maior ou menor risco identificado pelo questionário. Ao dividirmos a amostra entre aqueles que não referiram hospitalização nos últimos 12 meses e aqueles que reportaram pelo menos uma internação, encontramos um risco cerca de 3,5 vezes maior para o segundo grupo (RR 3,42; IC 95\%: 2,48-4,72; c2 Mantel-Haenszel p < 0,00001).

\section{Discussão dos resultados}

Os resultados obtidos por pela análise preliminar do instrumento apontam para sua utilidade na organização da porta de entrada e na estruturação das filas de acesso aos serviços de saúde, como um elemento que fornece informações sobre prioridades de atendimento. Nossos resultados se mostraram consistentes com achados de outros estudos (Boult et al., 1994) referentes ao risco de comprometimento do estado de saúde dos idosos.

Conforme mostrado na Figura 3, a maioria dos usuários, mesmo tendo sido captados no guichê de entrada de um serviço de saúde, apresenta risco baixo, tornando possível o planejamento de intervenções, de forma a mantê-los saudáveis, ativamente engajados no convívio social e permitindo que aqueles em maior risco sejam atendidos de forma prioritária.

O questionário proposto mostrou-se um instrumento eficiente para organizar e ordenar um serviço de saúde, definindo com clareza os idosos em maior risco e, portanto, que necessitam de atenção diferenciada e direcionada para a manutenção de suas capacidades funcionais, preservando e reabilitando sua condição de saúde. Isto não significa esquecer aqueles em menor risco: eles podem ter o cuidado à saúde desenvolvido em outras estruturas de atendimento, como os centros de convivência (Camargo \& Veras, 1995).

\section{Recomendações e conclusões}

O objetivo deste trabalho foi mostrar que o envelhecimento saudável deve não só fazer parte das preocupações do setor saúde, mas também ser incluída como prioridade na agenda social do país.

Um dos estrangulamentos dos sistemas de saúde é a captação eficiente dos que mais necessitam de cuidado, no momento certo.

Os resultados da análise dos dados transversais do trabalho fornecem evidência preliminar da eficácia do instrumento utilizado para classificar os idosos em diferentes grupos de risco de fragilização, possibilitando a hierarquização da atenção aos indivíduos. Futuros trabalhos deverão ser conduzidos, em um estudo prospectivo, visando a testar a eficiência do ins- 
Tabela 3

Distribuição dos fatores de risco, estratificados por níveis de risco, em uma amostra de idosos do Rio de Janeiro, Brasil, 2000.

\begin{tabular}{|c|c|c|c|c|c|c|c|c|}
\hline \multirow{2}{*}{ Fator de riscoa } & \multicolumn{2}{|c|}{ Risco 0 (baixo) } & \multicolumn{2}{|c|}{ Risco 1 (médio) } & \multicolumn{2}{|c|}{ Risco 2 (médio-alto) } & \multicolumn{2}{|c|}{ Risco 3 (alto) } \\
\hline & $\mathrm{n}$ & $\%$ & $\mathrm{n}$ & $\%$ & $\mathrm{n}$ & $\%$ & $\mathrm{n}$ & $\%$ \\
\hline \multicolumn{9}{|c|}{ Autopercepção da saúde } \\
\hline Excelente & 17 & 6,2 & 0 & & 0 & & 0 & \\
\hline Muito boa & 13 & 4,8 & 0 & & 0 & & 0 & \\
\hline Boa & 61 & 22,3 & 1 & 2,1 & 0 & & 0 & \\
\hline Média & 149 & 54,6 & 18 & 37,5 & 14 & 48,3 & 2 & 20,0 \\
\hline Ruim & 33 & 12,1 & 29 & 60,4 & 15 & 51,7 & 8 & 80,0 \\
\hline \multicolumn{9}{|l|}{ Hospitalizaçãob } \\
\hline Nenhuma vez & 255 & 93,4 & 34 & 70,8 & 21 & 72,4 & 2 & 20,0 \\
\hline $1 \mathrm{vez}$ & 12 & 4,4 & 9 & 18,8 & 4 & 13,5 & 4 & 40,0 \\
\hline 2 ou 3 vezes & 5 & 1,8 & 5 & 10,4 & 1 & 3,4 & 3 & 30,0 \\
\hline Mais de 3 vezes & 1 & 0,4 & 0 & & 3 & 10,3 & 1 & 10,0 \\
\hline \multicolumn{9}{|c|}{ Consultas médicasc } \\
\hline Nenhuma vez & 23 & 8,4 & 1 & 2,1 & 1 & 3,4 & 1 & 10,0 \\
\hline $1 \mathrm{vez}$ & 38 & 13,9 & 6 & 12,5 & 2 & 6,9 & & 0 \\
\hline 2 ou 3 vezes & 65 & 23,8 & 6 & 12,5 & 5 & 17,2 & 1 & 10,0 \\
\hline 4 a 6 vezes & 68 & 24,9 & 10 & 20,8 & 3 & 10,3 & 0 & \\
\hline Mais de 6 vezes & 79 & 28,9 & 25 & 52,1 & 18 & 62,1 & 8 & 80,0 \\
\hline \multicolumn{9}{|l|}{ Diabetes mellitus } \\
\hline $\operatorname{Sim}$ & 45 & 16,5 & 21 & 43,8 & 14 & 48,3 & 9 & 90,0 \\
\hline \multicolumn{9}{|c|}{ Doença cardiovasculard } \\
\hline Sim & 67 & 24,5 & 30 & 62,5 & 23 & 79,3 & 10 & 100,0 \\
\hline \multicolumn{9}{|l|}{ Sexo } \\
\hline Masculino & 92 & 37,7 & 16 & 33,3 & 14 & 48,3 & 4 & 40,0 \\
\hline Feminino & 181 & 66,3 & 32 & 66,7 & 15 & 51,7 & 6 & 60,0 \\
\hline \multicolumn{9}{|l|}{ Apoio sociale } \\
\hline Sim & 248 & 90,8 & 32 & 66,7 & 5 & 17,2 & 5 & 50,0 \\
\hline \multicolumn{9}{|l|}{ Idade } \\
\hline $65-74$ anos & 192 & 70,3 & 23 & 47,9 & 19 & 65,5 & 4 & 40,0 \\
\hline 75-79 anos & 55 & 20,1 & 16 & 33,3 & 7 & 24,1 & 4 & 40,0 \\
\hline $80-84$ anos & 22 & 8,1 & 5 & 10,4 & 2 & 6,9 & 1 & 10,0 \\
\hline$\geq 85$ anos & 4 & 1,5 & 4 & 8,4 & 1 & 3,4 & 1 & 10,0 \\
\hline
\end{tabular}

a Risco calculado segundo: Boult et al. (1993, 1994), Pacala et al. (1995).

b Hospitalização nos 12 meses anteriores à entrevista.

c Número de consultas médicas nos 12 meses anteriores à entrevista.

d Doença cardiovascular: angina, infarto do miocárdio, doença coronariana.

e Ajuda de amigo, parente ou vizinho, em caso de necessidade. 
Figura 3

Risco de comprometimento da saúde em uma amostra de idosos.

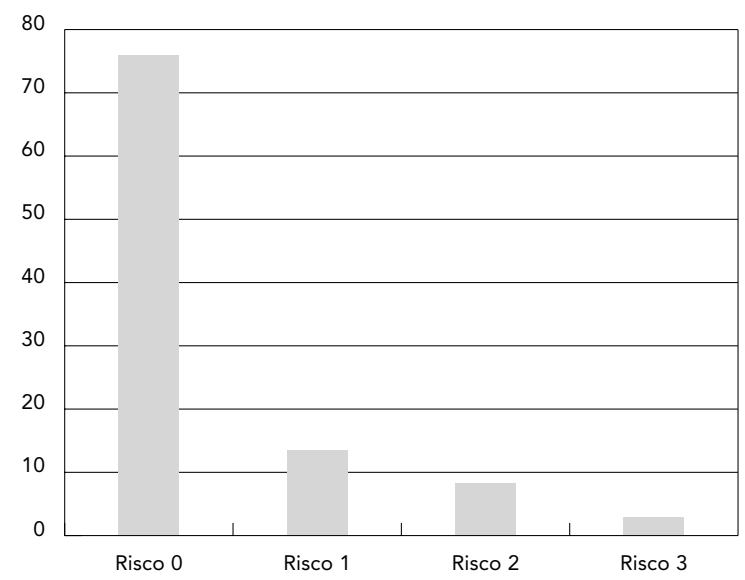

trumento, uma vez que o valor preditivo do "risco de fragilização" não é adequadamente identificado com o desenho do estudo aqui apresentado. Também a validação deve ser testada em outras populações idosas, pois no presente estudo foi assumido como similar ao identificado pelos autores da pesquisa original (Boult et al., 1993).

Este estudo procurou contribuir para a organização de um sistema de saúde mais eficaz e resolutivo para a população idosa. Para tal, foram empregadas ferramentas da Saúde Coletiva: Epidemiologia e Planejamento em Saúde.

\section{Agradecimentos}

Trabalho elaborado na Policlínica Piquet Carneiro, da Universidade do Estado do Rio de Janeiro (UERJ). Este trabalho contou com a colaboração e a participação dos seguintes professores e pesquisadores da Universidade Aberta da Terceira Idade/UERJ: Roberto Lourenço, Cláudia S. F. Martins, Maria Angélica S. Sanchez e Paulo Henrique Chaves.
Com a utilização desse instrumental, buscouse reforçar a tese, apresentada neste trabalho, da complexidade atual do setor saúde, que na conjuntura vigente exige soluções inovadoras e criativas.

O modelo aqui apresentado e testado reformata a estrutura do sistema de porta de entrada até então utilizado nas unidades de saúde. A partir do novo modelo, o idoso é captado, avaliado e referenciado adequadamente, permitindo uma melhor organização do atendimento e um manejo mais adequado das filas, tão comuns nas unidades de saúde.

A utilização de um instrumento específico para a identificação de risco permite priorizar o atendimento dos que mais necessitam de um serviço geriátrico com suporte interdisciplinar, promovendo a reabilitação e diminuindo os riscos de hospitalização (Guerra, 2002). O instrumento mostrou-se eficaz no reconhecimento do idoso em maior risco, que, portanto, deve ser olhado de forma prioritária pelos serviços assistenciais. A sua vantagem adicional é o fato de se tratar de um instrumento de fácil utilização, aplicado no momento da entrevista de admissão. Por sua construção, com perguntas simples e objetivas, permite que qualquer pessoa treinada o aplique em curto espaço de tempo e em diferentes locais.

A amostra deste trabalho comportou-se de forma bastante semelhante à de outros estudos sobre envelhecimento, em populações distintas (Boult et al., 1993, 1994), nas quais uma pequena proporção de idosos (em nosso caso, 19,5\%) apresentava risco significativo de fragilização, sugerindo ser esse um instrumento robusto, a despeito das limitações com relação à validade externa de quaisquer estudos com amostras de conveniência.

\section{Referências}

ALVES, M. I., 1997. Evolução da Mortalidade da População Idosa no Município do Rio de Janeiro. Dissertação de Mestrado, Rio de Janeiro: Instituto de Medicina Social, Universidade do Estado do Rio de Janeiro.

BOULT, C.; DOWD, B.; McCAFFREY, D.; BOULT, L.; HERNANDEZ, R. \& KRULEWITCH, H., 1993. Screening elders for risk of hospital admission. Journal of the American Geriatric Society, 41:811-817.

BOULT, C.; BOULT, L.; MURPHY, C.; EBBITT, B.; LUPTAK, M. \& KANE, R. L., 1994. A controlled trial of outpatient geriatric evaluation and management. Journal of the American Geriatric Society, 42:465470 . 
BRASIL, 1996. Decreto no 1.948, de 3 de julho de 1996. Regulamenta a Lei 8.842, sancionada em 4 de janeiro de 1994, a qual "dispõe sobre a Política Nacional do Idoso, cria o Conselho Nacional do Idoso e dá outras providências". Brasília: Diário Oficial da República Federativa do Brasil, vol. 134, no 128, p. 12277-12279, 3 jul. Seção 1.

BRASIL, 2002. Portaria no 249, de 16 de abril de 2002, aprovar na forma do Anexo 1 desta portaria as normas de cadastramento de centro de referência em assistência à saúde do idoso. 12 Agosto 2002 <http://www.saude.gov.br/sas/PORTARIAS/ PORT2002/PT-249/htm>.

CAMARANO, A. A. (org.), 1999a. Como Vive o Idoso Brasileiro? Rio de Janeiro: Instituto de Pesquisa Econômica Aplicada.

CAMARANO, A. A. (org.), 1999b. Muito Além dos 60: Os Novos Idosos Brasileiros. Rio de Janeiro: Instituto de Pesquisa Econômica Aplicada.

CAMARGO, K. \& VERAS, R. P., 1995. Idosos e universidade: parceria para qualidade de vida. In: Terceira Idade: Um Envelhecimento Digno para o Cidadão do Futuro (R. P. Veras, org.), pp. 11-27, Rio de Janeiro: Relume-Dumará/Universidade Aberta da Terceira Idade, Universidade do Estado do Rio de Janeiro.

FRIES, J. F., 1980. Aging, natural death and the compression of mortality. New England Journal of Medicine, 303:130-135.

FRIES, J. F. \& CRAPO, L. M., 1981. Vitality and Aging: Implications of the Rectangular Curve. San Francisco: WH Freeman and Company.

GORDILHO, A.; NASCIMENTO, J. S.; RAMOS, L. R.; FREIRE, M. P. A.; ESPINDOLA, N.; MAIA, R.; VERAS, R. \& KARSCH, U., 2000. Desafios a Serem Enfrentados no Terceiro Milênio pelo Setor Saúde na Atenção Integral ao Idoso. Rio de Janeiro: Universidade Aberta da Terceira Idade, Universidade do Estado do Rio de Janeiro.

GRUENBERG, L.; TOMPKINS, C. \& PORELL, F., 1989. The health status and utilization patterns of the elderly: Implications for setting medicare payments to HMOs. Advanced Health Economics \& Health Services Research, 10:41-73.

GUERRA, H. L., 2002. Internações Hospitalares entre Idosos: O Episódio da Clínica Santa Genoveva e Fatores Associados à Ocorrência de Internações em Bambuí, Minas Gerais. Tese de Doutorado, Rio de Janeiro: Escola Nacional de Saúde Pública, Fundação Oswaldo Cruz.

IBGE (Fundação Instituto Brasileiro de Geografia e Estatística), 2000. PNAD (Pesquisa Nacional por Amostra de Domicílio) Acesso e Utilização de Serviços de Saúde 1998. Rio de Janeiro: IBGE.

MATOS, A. J., 2000. Na contra-mão: O Brasil tem excesso - E não falta - De hospitais. Revista Exame, 720: 170.

MENDES, W., 2001. Home Care: Uma Modalidade de Assistência à Saúde. Rio de Janeiro: Universidade Aberta da Terceira Idade.

MOSEGUI, G. B.; ROZENFELD, S.; VERAS, R. P. \& VIANNA, C. M., 1999. Avaliação da qualidade do uso de medicamentos em idosos. Revista de Saúde Pública, 33:437-444.

O GLOBO, 2000. Joelmir Beting. O Globo, Rio de Janeiro, 13 ago. Primeiro Caderno, p. 34.
PACALA, J. T.; BOULT, C. \& BOULT, L., 1995. Predictive validity of a questionnaire that identifies older persons at risk for hospital admission. Journal of the American Geriatric Society, 43:374-377.

RAMOS, L. R., 1993. A explosão demográfica da terceira idade no Brasil: Uma questão de saúde pública. Gerontologia, 1:3-8.

ROZENFELD, S., 1997. Reações Adversas aos Medicamentos na Terceira Idade: As Quedas em Mulheres como Iatrogenia Farmacoterapêutica. Tese de Doutorado, Rio de Janeiro: Instituto de Medicina Social, Universidade do Estado do Rio de Janeiro.

SAYD, J. D.; FIGUEREDO, M. C. \& VAENA, M. L., 2001. Automedicação na população idosa no núcleo de atenção ao idoso na UnATI/UERJ. In: Velhice numa Perspectiva de Futuro Saudável (R. P. Veras, org.), pp. 115-134, Rio de Janeiro: Relume Dumará/Universidade Aberta da Terceira Idade, Universidade do Estado do Rio de Janeiro.

SILVESTRE, J.; KALACHE, A.; RAMOS, L. R. \&VERAS, R. P., 1998. Population ageing in Brazil and the health care sector. Bold: Quarterly Journal of The International Institute of Ageing, 7:4-12.

VERAS, R. P., 1994. País Jovem com Cabelos Brancos: A Saúde do Idoso no Brasil. Rio de Janeiro: Relume-Dumará/Universidade do Estado do Rio de Janeiro.

VERAS, R. P., 2000. O Anacronismo dos Modelos Assistenciais na Área da Saúde: Mudar e Inovar, Desafios para o Setor Público e o Privado. Estudos em Saúde Coletiva 211. Rio de Janeiro: Instituto de Medicina Social, Universidade do Estado do Rio de Janeiro.

VERAS, R. P., 2001. Modelos contemporâneos no cuidado à saúde: Novos desafios em decorrência da mudança do perfil epidemiológico da população brasileira. Revista USP, 51:72-85.

VERAS, R. P., 2002. Terceira Idade: Gestão Contemporânea em Saúde. Rio de Janeiro: Relume-Dumará.

VERAS, R. P. \& ALVES, M. I. C., 1994. A população idosa no Brasil: Considerações acerca do uso de indicadores de saúde, In: Os Muitos Brasis: Saúde e População na Década de 80 (M. C. S. Minayo, org.), pp. 320-337, São Paulo: Editora Hucitec.

VERAS, R. P.; LOURENÇO, R.; MARTINS, C. S. F.; SANCHEZ, M. A. \& CHAVES, P. H., 2002. Novos paradigmas do modelo assistencial no setor saúde: Conseqüência da explosão populacional dos idosos no Brasil. Medicina Social. In: Terceira Idade: Gestão Contemporânea em Saúde (R. Veras, org.), pp. 11-79, Rio de Janeiro: Universidade Aberta da Terceira Idade, Universidade do Estado do Rio de Janeiro/Relume Dumará.

VILAR, J. M., 2000. A crise no setor saúde e o ranking da OMS. Revista Brasileira de Home Care, 6:22.

Recebido em 13 de maio de 2002

Versão final reapresentada em 27 de julho de 2002

Aprovado em 5 de agosto de 2002 